\title{
An Ethnography on Joys and Sorrows of Women Undergoing Infertility Treatment in Korea
}

\author{
Seong-Heui Kim \\ Assistant Professor, Dept. of Nursing, Daejin Univ., Hoguk-ro 1007, Pocheon-si, \\ Gyeonggi-do, 11159, Republic of Korea \\ nursingkim@daejin.ac.kr
}

\begin{abstract}
This study aims to explore and understand experiences of women in Korea, who wanted children, but could not easily have them. It was motivated by the need to develop an effective nursing intervention for women undergoing infertility treatment under Korean sociocultural background. The informants consisted of nine married women who are diagnosed infertile and pursuing medical intervention. This study collected data through in-depth interviews and participant observations. The ethnographical analysis method, proposed by Spradley, was used to analyze these data. Consequently, infertility treatment was experienced as "getting rid of stigma of deficient women". This cultural theme can be divided into the following four categories: 'turning into life of a loser', 'doing homework to be recognized for my existence', 'the ambivalence about infertility treatment', 'positioning the value of being myself'. It was suggested that not only nursing in hospitals but also government's policy and social supports should be operated for infertility problem from the women's point of views in order to promote physical, mental, and socio-cultural health of women who are undergoing infertility treatment in Korean society, nursing studies should pay attend.
\end{abstract}

Keywords: Infertility, Women, Experience, Ethnography

\section{Introduction}

Since August 2019, South Korea has become a country in which a woman never has one child in her life. The nation's total fertility rate is less than 1 . That is 0 people (0.92). This is the lowest among OECD member countries [1][2]. The number of infertile couples are rapidly increasing in Korean society for a variety of reasons, including increased marriage age, stress, environmental pollution, number of working couples, etc. [1][3].

Recently, infertility treatment in Korea has been highly technical. And as the low birth rate has treated as a national crisis, the Korean government is actively pursuing support programs for infertile couples [1][4]. As the burden of expensive infertility treatment is reduced, infertility treatment procedures are increasing and becoming popular [5].

On the other hand, in the socio-cultural context of Korea, married women are given a position and role as the subject of childbirth. And still, the idea, that the essence of femininity is 'birth and raising a child', is internalized to the public [5].

In Korean society, where child-centered values are more prevalent than couple-centered values, social relationships including families are being formed through the mediation of

Article history:

Received (April 8, 2020), Review Result (May 12, 2020), Accepted (June 16, 2020) 
children [6]. So, the birth of a child is a big socio-cultural event that strengthens the bonds between couples and family cohesion. If a married woman does not become pregnant within a year, the couple are judged infertile [2][7]. And, infertile women are perceived as 'abnormal being'. As soon as they are judged as infertile women, they are encouraged and pressured by the hospitals and their families to treat infertility [8][9]. In the present situation, where infertility treatment is strongly supported at the national level, it has become a natural trend for infertile women to accept infertility treatment [10][11].

It is predicted that infertile women are undergoing a lot of physical pain and psychological stress due to difficult tests and long-term treatment. In particular, unlike couples who intentionally avoid childbirth because they want to enjoy their own lives, the women, who choose infertility treatment, have a strong will and hope the outcome of pregnancy and childbirth whether their choices are voluntary or pressure from others. It is reported that they have a lot of psychological, social and economic stress while undergoing repeated pregnancy failures during the treatment process [9][12].

With the combination of child-centered family culture in Korea, technological advances in infertility treatment and the Korean government's childbirth support policy, number of the women receiving infertility treatment are rapidly increasing in Korean society. Domestically and internationally, researches on infertility have been mainly pursued in the fields of medicine, psychology, and religion [9][13]. There are lack of researches in the field of nursing studies regarding experiences of women undergoing infertility from an emic perspective. And even a handful of nursing studies related to infertility are mostly survey studies using questionnaires, which are limited to in-depth understanding of the overall problems experienced by infertile women [14].

Since human reactions are formed in the culture to which they belong, the experiences of the subjects need to be understood in the socio-cultural context. In order to identify the cultural patterns shared by groups of women receiving infertility treatment, it is appropriate to apply an ethnographic research method [15].

Within the cultural background of Korean society, where social expectations or norms for traditional femininity still exist, it is very important to understand experiences of women undergoing infertility treatment from their point of view.

Therefore, this study aims to explore and understand joys and sorrows of women undergoing infertility treatment in Korean sociocultural background, and finally provide basic data for developing an effective nursing intervention for women to properly perform their gender role. The main research question of this study is "What are the experiences of women undergoing infertility treatment in Korean society?"

\section{Research method}

\subsection{Research design}

This is a qualitative research, using ethnographic research method that leads to the understanding of a cultural group by entering into their daily lives and describing and analyzing their experiences from their point of view.

\subsection{Selecting research field and informant}

The research field of this study is the infertility treatment center of two women's hospital where this researcher is guiding students for nursing practice. It is regarded, therefore, as the 
best place in terms of accessibility of the study, feasibility for permission, and possibility of participation.

The Informants are 9 women undergoing infertility treatment at the hospital. Informants were selected in a manner of opportunistic, maximum variation and theoretical sampling, as the data were being collected and analyzed, in on-going process. This researcher explained the aim and method of the study to the head nurse, who introduced appropriate informants, having over 10 years working experience at the hospital

Informants are 1 in their 20s, 7 in their 30s and 1 in their 40s.

The duration infertility treatment ranged from 16 months to 6 years.

\subsection{Data collection and ethical consideration}

The data were collected during the period of August to December 2019 in the manner of ethnographic interviews and participant observation

The in-depth interview was conducted at the counseling room in infertility center. Interviews in the beginning were conducted in descriptive question to have outline of and search domain of the experience, and then gradually structural and contrast questions are followed in order to listen and observe what she has in her mind.

The main interview questions were:

"How did infertility treatment begin?"

"Could you tell me about your daily life during infertility treatment?

"What are difficult things during infertility treatment?"

"Could you tell me your opinion about infertility treatment?"

Interview information was recorded by an mobile phone under the consent of the informant, and after finishing the interview process, the recordings and notes were transcribed and confirmed authenticity by this researcher as soon as possible. Informants were interviewed twice or three times for 2 or 3 hours for each interview.

Participant observations were conducted during interviews of the informants. It is also observed at informants' daily spot - treatment ward.

The data collection was done up to the saturation point, when new materials could no longer be found.

For ethical reasons, the data collection began after this researcher informed the informants about the spontaneity of research, the guarantee of the confidentiality of the research contents and utilization of the data for research purpose only, and then, accepted agreement of the informants to participate in this research.

\subsection{Data analysis}

The data in this study were analyzed according to Spradley's ethnographic analysis method.

This approach has a series of phases to discover and interpret the category, property, and cultural theme, from the various social situations through the process of domain, taxonomic analysis, componential analysis, and theme analysis. Exploring the cultural themes and meanings conducted in the last step is the core of an ethnography research which confirms cognitive principle repeatedly appearing in a culture and finds the relationship between each part and the whole of culture.

Data analysis was done not by one-time process after completing all of the data collection, but by repeating process of disintegration, integration, comparison, modification, interpretation, and re- interpretation of participant observation and collected interview data [15]. As a result, the cultural themes and meanings in this study were found 


\section{Results and discussion}

Using ethnographic research method, as the result of exploring patterns of experiences on the joys and sorrows of women undergoing infertility treatment in Korean society and analyzing the cultural meanings, "getting rid of stigma of deficient women" was deduced as the cultural theme.

Cultural theme has meaning structure of 4 categories and 14 properties.

The relationship between the cultural theme, categories, and properties is shown in below.

4 Categories are 'Turning into life of a loser', 'doing homework to be recognized for my existence', 'the ambivalence about infertility treatment', 'positioning the value of being myself'.

The identified properties in each category are as follows.

\subsection{Turning into life of a loser}

The category "Turning into life of a loser" has 2 properties: "Recommendations and pressures on normal family', 'Feeling shabby as an incomplete being'. This category shows experiences that participants are naturally forced to become pregnant after marriage, and at the same time, they gradually loses self-esteem beyond the problem of not getting pregnant in a social atmosphere that infertility women are not normal.

\subsubsection{Recommendations and pressures on normal family}

Not only did most of the informants suffer from the pressure that they should have a baby as soon as they get married, they also said that it became an atmosphere that gave them responsibility when they became pregnant over time. They said that Korea is a country where pregnancy is a mandatory course after marriage. In Korean society, children has traditionally been recognized as an element that strengthens the bonds between couples and family cohesion. So the common belief that married couples have to have children, and then they finally become a normal family, is naturally being infused into married couples.

\subsubsection{Feeling shabby as an incomplete being}

Informers have become infertile women naturally over a year after getting married as prescribed by modern medicine. They felt shameful, humble and desperate as if they were a loser in their lives, recognizing themselves as lacking being. Because they thought they would not be able to do this natural, normal process like giving a birth, building a family and raising children. It shows that the birth of children is becoming an event that gives women a sense of achievement and meaning of life, because the role of women is still considered to be the same as that of mothers in Korean society.

\subsection{Doing homework to be recognized for my existence}

The category "Doing homework to be recognized for my existence" has 4 properties :

'Living up to the expectations of family members', 'Trying to protect my position as wife',

'Looking for an imposing but ordinary woman's life', 'Expectations and relying on modern medical technology'.

\subsubsection{Living up to the expectations of family members}


The informants came to accept that in order to play the role assigned to them in the family, they had to take the responsibility for being pregnant and to adapt to infertility treatment for it. They said that they had chosen a way to be a good daughter-in-law and a good daughter in their family. This property shows that informants also have no choice but to be influenced by Korean traditional culture, which places more emphasis on parent-child relationships than marital relationships in family relationships.

\subsubsection{Trying to protect my position as wife}

This property reveals the experiences of informants choosing fertility treatment and going through a difficult course of treatment as a way to maintain their relationship with their husbands, express their love for their husbands, and act as roles and responsibilities as a wife.

\subsubsection{Looking for an imposing but ordinary woman's life}

This property is an experience in which infertile women continue treatment with the desire to become 'normal' and 'ordinary' women like other women, with feelings of shrinking about their own situation of infertility not only in family relationships and marital relationships but also in social relationships. In combination with work life and infertility treatment, they have always been subject to exceptions, and even the consideration of work for them has become a more uncomfortable experience. In addition, they realized that in Korea, if they had no experience in childbirth and parenting, they would not be treated as mature adults even if they were married.

\subsubsection{Expectations and relying on modern medical technology}

This property shows that women with infertility will come with great expectation and willingness to infertility treatment in an environment in which the legitimacy and justification of infertility treatment are secured with enormous support from the state.

\subsection{The ambivalence about infertility treatment}

The category " The ambivalence about infertility treatment " has 5 properties : 'All my daily routine focuses on pregnancy', 'Infertility treatment that provides comfort and hope', 'My body and mind becoming desolate', 'The discomfort for going against nature', 'Endless treatment like being in a swamp'.

\subsubsection{All my daily routine focuses on pregnancy}

This property demonstrates that, during infertility treatment, informants live a life for pregnancy only, lead a treatment-oriented life, and eventually have a skepticism and conflict about treatment as they feel that they are losing their lives.

\subsubsection{Infertility treatment that provides comfort and hope}

This property shows that formants, who have been confused and despaired by the diagnosis of infertility, experience the comfort and stability of the mind with confidence and hope that they can become pregnant through fertility treatment.

\subsubsection{My body and mind becoming desolate}


This property reveals that infertile women experience psychological devastation due to repeated pregnancy failures as well as physical pain due to invasive procedures and side effects of treatment drugs. During the infertility treatment process, strict schedules, invasive procedures, and intensive treatment are required for informants. In addition, they suffers physical pain from ovarian hyperstimulation syndrome, abdominal pain, and ascites.

\subsubsection{The discomfort for going against nature}

This property shows that informants continue to struggle due to the antipathy of artificial pregnancy and the unethical problems still being discussed during the process of infertility treatment. In particular, the fear of spoiling the natural flow of the body and forcibly making many ovum at once, and the antipathy of couple sex that were instrumentalized for pregnancy became a factor in strengthening the conflict over treatment.

\subsubsection{Endless treatment like being in a swamp}

This property shows the experience of being skeptical and anxious as providers become weary of days of undetermined treatment that endure with a vague dream of becoming a mother. They said that the longer the period of infertility treatment, the more loss of control over childbirth and the more difficult it was to decide when to stop treatment. The treatment process requires a long time and economic burden. Infertility treatment does not necessarily guarantee pregnancy, but it was not easy to stop once treatment started because it gives vague hope that one day will succeed.

\subsection{Positioning the value of being myself}

The category "Positioning the value of being myself" has 3 properties: "Expanding awareness of children and marriage', 'Accepting values centered on marital relationships', 'Strengthen my will to re-challenge for treatment'.

\subsubsection{Expanding awareness of children and marriage}

This attribute means that the subjects take time to look back on themselves as they experience several attempts and failures of pregnancy, and deviate a little from the obsession with pregnancy. In other words, this shows that they are willing to wait for pregnancy with a relaxed mind or expand their awareness of children and marriage.

\subsubsection{Accepting values centered on marital relationships}

This property means that infertile women are newly prepared to establish a new couplecentered value rather than a child-centered one and to cherish life with their husband.

\subsubsection{Strengthen my will to re-challenge for treatment}

This property demonstrates the characteristics of infertile women who endlessly challenge and do their best with the determination to maintain a traditional attitude of emphasizing children and to actively and consistently maintain treatment for pregnancy.

\section{Conclusion}


The number of women receiving infertility treatment is rapidly increasing due to mainly three factors, the child-centered family culture, technological advances in infertility treatment, and the Korean government's childbirth support policy. This study used an ethnographic research method to deeply explore experiences of women undergoing infertility treatment in the Korean socio-cultural context, and to understand the patterns and meaning of the experiences of the women.

This study found that a common experience of women undergoing infertility treatment in Korean society was "getting rid of stigma of deficient women." This means the cognitive principle repeatedly appeared in the culture of women group receiving infertility treatment. Through this study, it was confirmed the perception, that the main role and responsibility of married women is childbirth and parenting, is still deeply rooted in modern Korean society. So that, infertile women experienced the feelings of being a loser in life, recognizing themselves as lacking being, 'not normal'.

In addition, it showed cultural patterns that they relied on fertility treatment while looking forward to the lives of ordinary women and expecting to be recognized for their existence in the family and society, but eventually they could not have normal lives.

It was revealed that infertile women had comfort and hope in treating infertility, but on the other hand, they had an ambivalent feeling about the treatment by experiencing all the daily routines centered on pregnancy, the devastation of the body and mind, treatment procedures that are going against nature, and the characteristics of treatment that are not guaranteed but cannot be stopped.

While experiencing repeated failures of pregnancy, women have the opportunity to expand their perception of children, or to accept marital-centered values rather than clinging to excessive expectations of infertility treatment. Otherwise, they redefined their will for new challenges while observing the success of pregnancy around them. Eventually, it represented cultural patterns that women are experiencing the mature process of establishing their own values.

Since this study was conducted on nine women receiving infertility treatment in two hospitals, it is limited to generalize the results of the study, even if the data collection reached saturation level.

\section{References}

[1] Statistics Korea, "Infertility experience of married women (15 49 years) and husbands", Statistic Korea 2019, http://kostat.go.kr/wnsearch/search.jsp, (2019)

[2] Y. S. Lee, S. M. Choi, and J. H. Kwon, "Psychosocial predictors of infertile women's distress," Journal of Korean Society of Maternal and Child Health, vol.23, no.2, pp.136-146, (2019)

[3] M. Kim, H. Nam, and M. Youn, "Infertility stress, depression, and resilience in women with infertility treatments," Journal of Korean Public Health Nursing, vol.30, no.1, pp.93-104, (2016)

[4] P. Kathleen, J. Debra, and R. Trudy, "Failures of reproduction; problematising 'Success' in assisted reproductive technology," Nursing Inquiry, vol.14, no.2, pp.125-131, (2007) DOI: 10.1111/j.14401800.2007.00363.x

[5] N. Hwang and I. Jang, "Factors influencing the depression level of couples participation in the national supporting program for infertile couples," Journal of Korean Community Nursing, vol.26, no.3, pp.179-189, (2005)

[6] S. H. Kim and E. K. Yoo, "Ethnographic research on adjustment of mothers caring for their cancer children in Korea," Korean Journal of Women Health Nursing, vol.21, no.3, pp.216-231, (2015) 
[7] K. Gourounti, F. Anagnostopoulos, and G. Vaslamatzis. "Psychosocial predictors of infertile related stress: a review," Current Women's Health Reviews, vol.6, no.4, pp.318-331, (2010)

[8] S. H. Kim, "A study of unmarried women's experiences on illegal abortion in south Korea," Asia Life Sciences Supplement, vol.19, no.2, pp.143-154, (2019)

[9] Mirian Ulrich and Ann Weatherall, "Motherhood and infertility: viewing motherhood through the lens of infertility," Feminism Psychology, vol.10, no.3, pp.323-336, (2000) DOI: 10.1177/0959353500010003003

[10] L. A. Pasch and K. T. Sullivan, "Stress and coping in couples facing infertility," Current Opinion in Psychology, vol.13, no.1, pp.131-135, (2017)

[11] J. W. M. Aart, V. Empel, J. Boivin, W. L. Nelen, J. A. M. Kremer, and C. M. Verhaak, "Relationship between quality of life and distress in infertility, a Validation Study of the Dutch FertiQoL," Human Reproduction, vol.26, no.5, pp.11124-1118, (2011)

[12] J. Boivin, J. E. Takefman, and A. Braverman, "The fertility quality of life tool: development and general psychometric properties," Human Reproduction, vol.26, no.8, pp.2084-2091, (2011)

[13] Cheryl de Lacey, "IVF as lottery or investment: contesting metaphors in discourses of infertility", Nursing Inquiry, vol.9, no.1, pp.43-51, (2002) DOI: 10.1046/j.1440-1800.2002.00126.x

[14] Catherine Waldby and Melinda Cooper, "The biopolitics of reproduction post-fordist biotechnology and women's clinical labour" Australian Feminist Studies, vol.23, no.55, pp.57-73, (2008) DOI: 10.1080/08164640701816223

[15] J. P. Spradley, “The ethnographic interview," Waveland Press, INC. Long Grove, Illinois, USA, pp.3-235, (2016) 